\title{
Research Article \\ Robust Control for Systems with Bounded-Sensor Faults
}

\author{
Chieh-Chuan Feng \\ Department of Electrical Engineering, I-Shou University, Kaohsiung City 84001, Taiwan \\ Correspondence should be addressed to Chieh-Chuan Feng, ccfeng@isu.edu.tw
}

Received 26 February 2012; Revised 4 May 2012; Accepted 15 May 2012

Academic Editor: Zhan Shu

Copyright (C) 2012 Chieh-Chuan Feng. This is an open access article distributed under the Creative Commons Attribution License, which permits unrestricted use, distribution, and reproduction in any medium, provided the original work is properly cited.

In this study an observer-based novel design of robust control system with an estimate scheme of sensor states to accommodate extended bounded-sensor faults is proposed. The sensor faults are, in general, modeled as polytopic bounds in robust control framework and are usually given as $a$ priori assumption. But, in practice, the sensors that are subject to faults are especially vulnerable to variant conditions, such as high temperature, and humidity. Thus, their faults may fall outside the presumed polytopic bounds easily. An estimate scheme of sensor states integrated into the observerbased control system captures the sensor faults outside the presumed region. The notion of quadratic stability stabilizes the overall system and guarantees a robust performance measure of an output error signal in the presence of a set of extended admissible sensor faults. A numerical example shows the effectiveness of the proposed approaches.

\section{Introduction}

The problems of designing fault-tolerant control systems have attracted considerable attention. Much efforts have gone to advancing practical usage of fault-tolerant systems within the avionics industry, see [1-4], for example. These high-risk applications require fault safe operation; that is, the system can withstand tolerable faults without effects on the system operations. Theoretical results and application examples being developed in the literature are, in general, divided into two broad spectrums: active [1, 5-8] and passive [2-4, 9-18]. In the active approach, reconfigurable mechanism has been designed in the event of bounded faults. Due to flexible capacity of the mechanism, the controller in such systems is not in a fixed form. This increases the complexity of the control design. Such fault-tolerant control systems use reconfigurable structure either online or predefined control law. [5] designed a pseud-inverse technique that maintained the closed-loop system as much as possible to the original system by reconfiguring the control law, once the faults encountered. [6] used 
eigenstructure assignment that was capable of recovering the nominal system after faults occurred. The controller was insensitive to the variation of devices $[7,8]$, have used direct adaptive control schemes to update control parameters and account for the case where the sensing devices were stuck at some fixed values. The advantages of active approaches are the use of reconfigurable or adaptive structure of online controller.

In contrast, the passive fault-tolerant control is to exploit the inherent redundancy of the system components or to use the remaining functions of the component to design a fixed compensator so as to achieve a tolerable system performance in the presence of component faults. The designed fixed controller guarantees satisfactory system performance not merely during normal operations, but under variant fault conditions. [9] tolerated faults by using linear quadratic state-feedback control for a prescribed set of actuators. A given performance bound was guaranteed. The controller of [10] based on robust pole region assignment method guaranteed stability and maintained an acceptable performance in the event of actuator faults. Taken as bounded measurement noises, sensor faults are estimated by fault estimation techniques in [15]. The proposed estimator accomplished the observation of system states and measurement noises simultaneously. [16] designed a fault estimation scheme for sensor with bounded time derivative of faults. Based on linear matrix inequality techniques, [2$4,11,12,17,18$ ] have designed robust controllers for sensor or actuator faults in the prescribed bounds with disturbance attenuation and satisfactory performance. In addition, $[17,18]$ treated sensor fault as a state of the system, which implies the possible boundedness of the faults. Notably, the aforementioned passive approaches have focused on the faults in a fixed bound and given as a priori information. To increase flexibility of passive approach, the advantages of active approach being adaptive are absorbed into passive design in this study.

This paper deals with extended bounded-sensor-faults, in which sensor faults may fall outside the presumed bounds in time varying or nonlinear manners. A passive form of observer-based controller with an integrated estimate scheme of sensor states captures the phenomena of extended bounded-sensor-faults. A basic idea of control design to extended faults of sensors in an observer-based control system relies on the plant states being correctly estimated under the corrupted measurement signals. To reach this goal, [11] designed a novel observer with an estimate scheme of sensor states in which the asymptotic stability of the system was restricted to against bounded and piece-wise constant faults. This result, however, is somewhat unrealistic. The study of this paper adopts the observer with estimate scheme idea as [11] but relaxes the piece-wise constant restriction to allow the sensor faults to be time-varying and/or nonlinear with bounds. Moreover, these presumed faulty sensors are allowed not to be in the presumed bounds. The observer with estimate scheme identifies these faulty conditions and computes the correct states for feedback. The notion of quadratic stability not only ensures the asymptotic stability of the system but also establishes $\mathcal{L}_{2}$-gain measure for the robust performance of a controlled error signal in the presence of a set of extended admissible sensor faults.

This paper is organized as follows. In Section 2, the system including observer structure and fault models of bounded piece-wise constant function is formulated. To proceed with Section 2, Section 3 gives the preliminary results developed in [11]. Section 4 presents the main results, which deal with the robust performance against extended boundedsensor faults and is divided into three subsections including system reformulation, quadratic stability and Linear matrix inequality (LMI) characterizations. Section 5 includes the synthesis of observer and control gains in terms of LMIs. Section 6 demonstrates effectiveness of proposed method by a numerical example. Last section, Section 7, concludes the overall results shown in the paper. 


\section{Problem Formulation}

Consider a linear time-invariant dynamical system with sensor faults

$$
\begin{gathered}
\dot{x}(t)=A x(t)+B u(t)+B_{1} d(t), \quad x(0)=x_{0}, \\
y(t)=C x(t), \\
y_{s}(t)=\operatorname{diag}[y(t)] \phi(t)=\operatorname{diag}[\phi(t)] y(t),
\end{gathered}
$$

where $x(t) \in \mathfrak{R}^{n}$ is the state vectors, and $u(t) \in \mathfrak{R}^{m}$ is the control signal of actuator. $y(t) \in \mathfrak{R}^{l}$ is the output of the system, and $y_{s}(t) \in \mathfrak{R}^{l}$ is the true measured output of sensor. $d(t) \in \mathfrak{R}^{d}$ is the disturbance. The representation of $\operatorname{diag}[y(t)]$, when the vector $y(t)$ is a vector with $l$ components, is a square matrix of dimension $l$ with the elements of $y(t)$ on the diagonal. The sensor function, $\phi(t) \in \mathfrak{R}^{l}$, is to represent the remaining function of the associated sensor. For example, if a sensor $\phi_{k}(t)=0.8$, in which $\phi_{k}(t)$ represents the remaining function of $k$ th sensor in the vector $\phi(t)$, then we say the sensor is $80 \%$ functioning.

Now, consider a state observer with control law of the following form:

$$
\begin{gathered}
\dot{\hat{x}}(t)=A \widehat{x}(t)+B u(t)+L\left(y_{s}(t)-\operatorname{diag}[\widehat{\phi}(t)] C \widehat{x}(t)\right), \\
u(t)=K \widehat{x}(t),
\end{gathered}
$$

where the vectors $\hat{x} \in \mathfrak{R}^{n}$ are the state of observer, which is an estimate of $x(t)$. Similarly, $\widehat{\phi}(t)$, an estimate of $\phi(t)$, which will be shown later in the sequel, is useful to observe the state $x(t)$ in the presence of sensor faults. $L$ and $K$ are the observer gain and control gain, respectively, to be designed such that the control objectives are achieved. Notice that the following expressions are interchangeable when later deriving the formulas, $\operatorname{diag}[C x(t)] \phi(t)=\operatorname{diag}[\phi(t)](C x(t))$ and $\operatorname{diag}[C \widehat{x}(t)] \phi(t)=\operatorname{diag}[\phi(t)](C \widehat{x}(t))$.

\section{Preliminaries}

The following assumptions are used for demonstrating the asymptotic stability based on Lyapunov method shown in the Theorem 3.1, which will be then relaxed while $\mathcal{L}_{2}$-gain robust performance is pursued in the next section.

(1) $\phi_{k}(t) \in[0,1]$,

(2) $\lim _{\Delta t \rightarrow 0}\left(\Delta \phi_{k}\left(t_{i}\right) / \Delta t\right)=0$, where $\Delta \phi_{k}\left(t_{i}\right)=\Delta \phi_{k}\left(t_{i}+\Delta t\right)-\Delta \phi_{k}\left(t_{i}\right)$, except at some time instants that $\phi_{k}(t)$ jump toward zero.

The assumptions addressed above have the following interpretations: $\phi_{k}(t)=0$ means the sensor fails. $\phi_{k}(t)=1$ means the sensor works properly. A fault sensor will be such that $0<\phi_{k}(t)<1$. Thus, it is a bounded sensor fault. The $\lim _{\Delta t \rightarrow 0}\left(\Delta \phi_{k}\left(t_{i}\right) / \Delta t\right)=0$ and $\phi_{k}(t)$ jumping toward zero mean that the sensor fault not only is a piecewise constant process but also indicate that $\phi_{k}(t)$ is a bounded above function. The analysis of asymptotic stability of the closed-loop system against bounded and piecewise constant sensor faults is first studied 
in [11], which is revealed in the following theorem and is stated for the completeness. We define the following sets:

$$
\mathbf{\Phi}=\left\{\operatorname{diag}[\phi] \mid \phi=\left(\phi_{1}^{T} \phi_{2}^{T} \cdots \phi_{l}^{T}\right)^{T}, \phi_{k} \in \mathfrak{R}, \phi_{k} \in\left[\underline{\phi}_{k^{\prime}} \bar{\phi}_{k}\right]\right\}
$$

and the vertex set of $\boldsymbol{\Phi}$ is defined as

$$
\boldsymbol{B} \boldsymbol{\Phi}=\left\{\operatorname{diag}[\phi] \mid \phi=\left(\phi_{1}^{T} \cdots \phi_{l}^{T}\right)^{T}, \phi_{k}=\underline{\phi}_{k} \text { or } \phi_{k}=\bar{\phi}_{k}\right\}
$$

Notice that it is easy to see that there are $2^{l}$ vertices in $\mathbf{B \Phi}$ to represent the possible faults in a known convex set.

Theorem 3.1. Assumptions 1 and 2 hold. Consider the system (2.1) and (2.2) for the case $B_{1}=0$ and $\operatorname{diag}[\phi] \in \mathbf{B \Phi}$.

If the following exist:

(1) the matrices $Q$ and L satisfying

$$
\begin{aligned}
& Q=Q^{T}>0, \\
& \Xi_{1}(Q)<0,
\end{aligned}
$$

where

$$
\Xi_{1}(Q)=(A-L(\operatorname{diag}[\phi]) C)^{T} Q+Q(A-L(\operatorname{diag}[\phi]) C)
$$

(2) the matrices $P$ and $K$ satisfying

$$
\begin{aligned}
& P=P^{T}>0, \\
& \Xi_{2}(P)<0,
\end{aligned}
$$

where

$$
\Xi_{2}(P)=(A+B K)^{T} P+P(A+B K),
$$

(3) for a given matrix $S>0$, the matrices $W>0$ and $\Gamma$ satisfying

$$
\begin{gathered}
\Gamma=\Gamma^{T}>0, \\
W+W^{T}-S>0,
\end{gathered}
$$


then the closed-loop system

$$
\begin{gathered}
\dot{x}=A x+B K \hat{x}, \\
\dot{\hat{x}}=(A+B K-L(\operatorname{diag}[\widehat{\phi}]) C) \widehat{x}+L y_{s}, \\
\dot{\hat{\phi}}= \begin{cases}\Gamma(\operatorname{diag}[C \widehat{x}]) L^{T} Q \tilde{x} & \text { for } \widehat{\phi} \in D \\
-\Gamma W \widehat{\phi}+\Gamma(\operatorname{diag}[C \widehat{x}]) L^{T} Q \tilde{x} & \text { for } \widehat{\phi} \in \bar{D}, \\
\dot{\tilde{x}}=A \tilde{x}-L(\operatorname{diag}[\widehat{\phi}]) C \widehat{x}+L y_{s}\end{cases}
\end{gathered}
$$

is asymptotically stable for $\widehat{\phi} \in D$, where $D$ is as follows:

$$
D=\left\{\widehat{\phi} \mid\|\hat{\phi}\|^{2} \leq \frac{\bar{\lambda} l}{\underline{\rho}}\right\}, \quad \bar{D}=\left\{\widehat{\phi} \mid\|\hat{\phi}\|^{2}>\frac{\bar{\lambda} l}{\underline{\rho}}\right\} .
$$

The state error is defined as $\tilde{x}=\hat{x}-x$. The parameters, $\bar{\lambda}$ and $\rho$, are the maximum and minimum eigenvalues of $W^{T} S^{-1} W$ and $W+W^{T}-S$, respectively, for some positive definite symmetric matrices $W$ and $S$ and $W+W^{T}-S>0$.

Proof. Refer to [11] for the proof.

Remark 3.2. The overall closed-loop system (3.8a)-(3.8d) decomposed into input-output structured block diagram in Figure 1, where the error filter (3.8d), the adaptive law (3.8c), and the state observer $(3.8 \mathrm{~b})$ form the augmented observer. Without sensor faults, the block diagram Figure 1 is simplified to Figure 2 where $\phi$ and $\widehat{\phi}$ are both identity matrices, and the block of adaptive law can be removed from Figure 1.

\section{Robust Performance}

In the last section the asymptotic stability, based on Lyapunov method, has been shown for the system with bounded sensor faults under piecewise constant assumption. Now, a set of extended sensor faults relax the previous restrictions to not only admit bounded time varying and/or nonlinear sensor function in the vertex set, $\boldsymbol{В \Phi}$, but also let the true sensor faults, which may fall outside the presumed bound, be norm bound. A closed-loop autonomous system will be reformulated in the following subsection. 


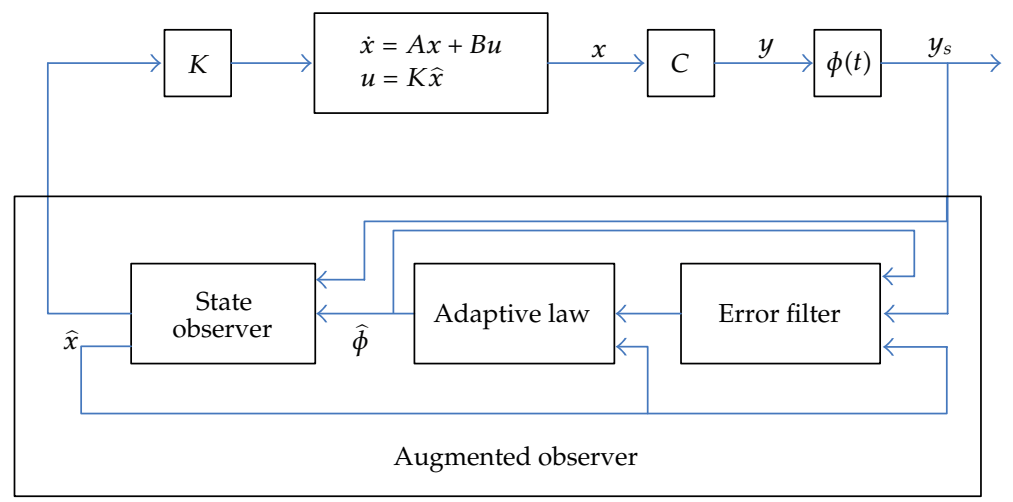

Figure 1: The input-output structured block diagram of the closed-loop system (3.8a)-(3.8d).

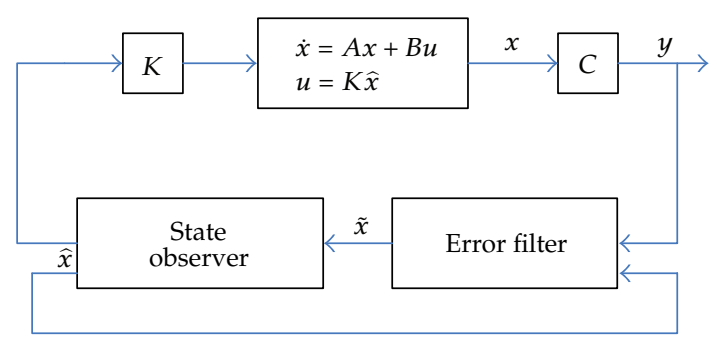

Figure 2: An ordinary observer-based control system.

\subsection{System Reformulation}

Consider closed-loop system (3.8a)-(3.8d), which can be rewritten as

$$
\begin{gathered}
\dot{x}_{c}=A_{c} x_{c}+B_{c} w, \quad x_{c}(0) \in \mathfrak{B}, \\
q=C_{q} x_{c}, \\
e=C_{e} x_{c}, \\
w=\operatorname{diag}[\tilde{\phi}] q,
\end{gathered}
$$

where

$$
\begin{gathered}
x_{c}=\left(\begin{array}{c}
\tilde{x} \\
x \\
\widehat{\phi}
\end{array}\right), \quad B_{c}=\left(\begin{array}{c}
-L \\
0 \\
0
\end{array}\right), \\
C_{q}=\left(\begin{array}{lll}
C & C & 0
\end{array}\right), \quad C_{e}=\left(\begin{array}{lll}
C & 0 & 0
\end{array}\right), \\
A_{c}=\left(\begin{array}{ccc}
A-L(\operatorname{diag}[\phi]) C & 0 & 0 \\
B K & A+B K & 0 \\
\Gamma(\operatorname{diag}[C \hat{x}]) L^{T} X_{1} & 0 & -\Gamma W
\end{array}\right),
\end{gathered}
$$


$\operatorname{diag}[\phi] \in \boldsymbol{B \Phi}$, and $\operatorname{diag}[\tilde{\phi}]=\operatorname{diag}\left(\tilde{\phi}_{1}, \ldots, \tilde{\phi}_{l}\right) . \boldsymbol{B} \subset \mathfrak{R}^{n}$ defines a domain that contains the origin. The matrices $K, L, \Gamma$, and $X_{1}$ are to be determined in the sequel. The signal, $w$, is a bounded exogenous signal that comprises $\tilde{\phi}$ and $C \hat{x}$, that is, $w=\operatorname{diag}[\tilde{\phi}] C \hat{x}$, where $\tilde{\phi}$ is the difference of estimated sensor function, $\widehat{\phi}$, and true sensor function, $\phi$, that is $\tilde{\phi}=\widehat{\phi}-\phi$. The estimation error $\tilde{\phi}(t)$ is assumed to belong to the following diagonal norm-bound set:

$$
\Delta \triangleq\left\{\tilde{\phi}_{i}(t)|| \tilde{\phi}_{i}(t) \mid \leq 1, i=1, \ldots, l\right\}
$$

which defines the extended sensor faults.

Remark 4.1. We have noticed that if the true sensor function falls within the presumed polytopic bound, $\boldsymbol{B} \Phi$, then the diagonal norm-bound set, which is confined within $\boldsymbol{\Delta}$, is partially (or maybe completely) overlaid with the $\boldsymbol{B} \Phi$. However, if the true sensor function does not fall into the set $\boldsymbol{B \Phi}$, then it is possible that $\boldsymbol{B \Phi}$ intersects $\Delta$ partially or their intersection is an empty set.

Remark 4.2. It is worth noting that, under the above assumption, the signal $w$ is such that

$$
w^{T} w=\widehat{x}^{T} C^{T}(\operatorname{diag}[\tilde{\phi}])^{T}(\operatorname{diag}[\tilde{\phi}]) C \widehat{x} \leq \widehat{x}^{T} C^{T} C \widehat{x},
$$

which for asymptotically stable systems can be bounded above and will be demonstrated in the sequel.

\subsection{Robust Performance Measure}

This subsection defines a robust performance measure and states an important theorem on which the robust performance is established. We assume that not all state information is available and is concerned with designing a fixed structure observer-based controller to stabilize the system (4.1) satisfying a given $\mathcal{L}_{2}$-gain constraint in response to all admissible sensor faults. The following notion of stabilizability for the system (4.1) with robust $\mathcal{L}_{2}$-gain measure is defined.

Definition 4.3. Let the constant $\gamma>0$ be given. The system (4.1) is said to be stabilizable with a robust $\mathcal{L}_{2}$-gain measure $\gamma$ if there exists a fixed control law $u=K \widehat{x}$ and observer gain $L$ such that for any admissible sensor faults the following conditions are satisfied.

(1) The system is uniformly asymptotically stable.

(2) Subject to the assumption of zero initial condition, the controlled output $e$ satisfies

$$
\frac{\|e\|_{2}}{\|w\|_{2}} \leq r
$$

Here, the quadratic stability with an $\mathcal{L}_{2}$-gain measure was introduced [19]. This concept is a generalization of that of quadratic stabilization to handle $\mathcal{L}_{2}$-gain measure constraint on exogenous attenuation. To this end, the characterizations of robust performance based on 
quadratic stability will be given in terms of matrix inequalities, where if LMIs can be found, then the computations by finite dimensional convex programming are efficient.

Theorem 4.4. Consider the closed-loop system (4.1), and the following statement holds: the closedloop system is said to be quadratically stable with a robust $\mathcal{L}_{2}$-gain measure $\gamma$ from input $w$ to output $e$ if there exists $X>0$ and $\lambda \geq 0$ such that

$$
\Pi<0,
$$

where

$$
\Pi=\left(\begin{array}{cc}
A_{c}^{T} X+X A_{c}+C_{e}^{T} C_{e}+\lambda C_{q}^{T} C_{q} & X B_{c} \\
B_{c}^{T} X & -\gamma^{2} I
\end{array}\right) .
$$

Proof. Let quadratic Lyapunov function be $V\left(x_{c}\right)=x_{c}^{T} X x_{c}^{T}$, with $X>0$ such that

$$
\begin{aligned}
& \forall x_{c} \text {, and } w \text { satisfying (4.1), } \\
& \frac{d}{d t} V\left(x_{c}\right)+e^{T} e-\gamma_{1}^{2} w^{T} w<0
\end{aligned}
$$

and constraint

$$
w^{T} w \leq x_{c}^{T} C_{q}^{T} C_{q} x_{c}
$$

Then, it follows from the $S$ procedure that the equivalent condition of (4.11) is the existence of $\lambda \geq 0$ satisfying

$$
\frac{d}{d t} V\left(x_{c}\right)+e^{T} e-r_{1}^{2} w^{T} w+\lambda\left(x_{c}^{T} C_{q}^{T} C_{q} x_{c}-w^{T} w\right) \leq 0,
$$

which can be equivalently written as

$$
\frac{d}{d t} V\left(x_{c}\right)+\lambda x_{c}^{T} C_{q}^{T} C_{q} x_{c}+e^{T} e-r^{2} w^{T} w \leq 0
$$

for $\gamma^{2}=\gamma_{1}^{2}+\lambda$. Then the $\mathcal{L}_{2}$-gain of the (4.1) is less than $\gamma$. To show this, we integrate (4.14) from 0 to $T$, with the initial condition $x_{c}(0)=0$, to get

$$
V\left(x_{c}(T)\right)+\lambda \int_{0}^{T} x_{c}^{T} C_{q}^{T} C_{q} x_{c} d t+\int_{0}^{T}\left(e^{T} e-r^{2} w^{T} w\right) d t \leq 0
$$

Since $V\left(x_{c}(T)\right)+\lambda \int_{0}^{T} x_{c}^{T} C_{q}^{T} C_{q} x_{c} d t \geq 0$, this implies that

$$
\frac{\|e\|_{2}}{\|w\|_{2}} \leq \gamma
$$


The inequality (4.8) and definition (4.9) are obtained by substituting (4.1) into (4.14). Without loss of generality, we will adopt only strict inequality in this paper. This completes the proof.

\subsection{Matrix Inequality Characterizations}

The following lemma is to show that the energy of the estimated output signals by observer can be limited by some matrix inequalities, which provide an upper bound of the exogenous signal, $w$.

Lemma 4.5. Given that

$$
\begin{gathered}
\dot{x}_{c}=A_{c} x_{c}, \quad x_{c}(0) \in \mathbb{B}, \\
\widehat{y}_{i}=C_{q, i} x_{c}, \\
B=\left\{x_{c} \mid x_{c}^{T} X x_{c} \leq v\right\}, \quad X>0, v>0,
\end{gathered}
$$

if there exist $X>0$ and $\theta_{i}>0$ satisfying

$$
\begin{gathered}
A_{c}^{T} X+X A_{c}<0, \\
\Upsilon_{i}>0, \quad i=1, \ldots, l,
\end{gathered}
$$

then the following statements are equivalent:

(1) $\widehat{y}_{i}^{2}<\left(\theta_{i} / v\right)$,

(2) $\widehat{\Theta}>0$, where

$$
\begin{gathered}
\Upsilon_{i}=\left(\begin{array}{cc}
X & C_{q, i}^{T} \\
C_{q, i} & \frac{\theta_{i}}{v} I
\end{array}\right), \\
\widehat{\Theta}=(\operatorname{diag}[C \widehat{x}])(-v I)(\operatorname{diag}[C \widehat{x}])+\Theta, \\
\Theta=\operatorname{diag}\left(\theta_{1}, \ldots, \theta_{l}\right) .
\end{gathered}
$$

Proof. $\mathrm{B}$ is an invariant ellipsoid. Let $V\left(x_{c}\right)=x_{c}^{T} X x_{c}$. Since

$$
\dot{V}\left(x_{c}\right)=x_{c}^{T}\left(A_{c}^{T} X+X A_{c}\right) x_{c}<0
$$

then we have

$$
V\left(x_{c}(t)\right) \leq V\left(x_{c}(0)\right) \leq v .
$$


Hence, $x_{c}(t) \in \mathbb{B}$, for all $t \geq 0$ and

$$
\widehat{y}_{i}^{2} \leq \max _{x_{c} \in \mathcal{B}} x_{c}^{T} C_{q, i}^{T} C_{q, i} x_{c}
$$

Let $\theta_{i}=\max _{x_{c} \in \mathcal{B}} x_{c}^{T} C_{q, i}^{T} C_{q, i} x_{c}$. We have

$$
\left(\begin{array}{cc}
X & C_{q, i}^{T} \\
C_{q, i} & \frac{\theta_{i}}{v} I
\end{array}\right)>0, \Longleftrightarrow C_{q, i}^{T} C_{q, i}<\frac{\theta_{i}}{v} X, \quad \forall i .
$$

From (4.23) and (4.24), we have

$$
\widehat{y}_{i}^{2} \leq \max _{x_{c} \in \mathcal{B}} x_{c}^{T} C_{q, i}^{T} C_{q, i} x_{c}<\frac{\theta_{i}}{v}, \quad \forall i
$$

This completes the proof of (1). The equivalence of (1) and (2) is straightforward.

Before stating the main theorem for the robust $\mathcal{L}_{2}$-gain measure $\gamma$ of the closed-loop system (4.1), which ensures the robust performance of the original system (2.1) and (2.2) against sensor faults, the following matrices are defined:

$$
\begin{aligned}
& \Pi_{1}=\left(\begin{array}{ccc}
\Xi_{2}\left(X_{2}\right)+\lambda C^{T} C & X_{2} B K+\lambda C^{T} C & 0 \\
(B K)^{T} X_{2}+\lambda C^{T} C & (\star) & -X_{1} L \\
0 & -L^{T} X_{1} & -\gamma^{2} I
\end{array}\right), \\
& \Pi_{2}=\left(\begin{array}{cc}
-(\Gamma W)^{T} X_{3}-X_{3}(\Gamma W)+2 \kappa X_{3} & X_{3} \Gamma \\
\Gamma^{T} X_{3} & -v I
\end{array}\right),
\end{aligned}
$$

where

$$
(\star)=\Xi_{1}\left(X_{1}\right)+(\lambda+1) C^{T} C+\left(X_{1} L\right) \Theta\left(L^{T} X_{1}\right) .
$$

Theorem 4.6. Let the $\gamma>0, \kappa>0$, and $v>0$ be given. The closed-loop system (4.1) with the admissible bounded sensor faults is said to be quadratically stable with a robust $\mathcal{L}_{2}$-gain measure $\gamma$, if one lets the matrix $X$ be in the set $X$

$$
x=\left\{\text { block } \operatorname{diag}\left(X_{1}, X_{2}, X_{3}\right) \mid X_{i}=X_{i}^{T}>0\right\},
$$

there exist $K, L, \Gamma>0, \Theta>0$, and $X \in X$ such that $\Pi_{1}<0, \Pi_{2}<0$, and $\Upsilon_{i}>0, i=1, \ldots, l$. 
Proof. We consider the signals $e(\cdot)$ in response to the signals $w(\cdot)$ with the zero initial states, and a (candidate) quadratic Lyapunov function $V\left(x_{c}\right) \triangleq x_{c}^{T} X x_{c}$. For any nonzero vectors, $\varsigma_{1}$ and $\varsigma_{2}$ are defined as

$$
\varsigma_{1}=\left(\begin{array}{c}
x \\
\tilde{x} \\
w
\end{array}\right), \quad \varsigma_{2}=\left(\begin{array}{c}
\widehat{\phi} \\
\operatorname{diag}[C \widehat{x}] L^{T} X_{1} \tilde{x}
\end{array}\right)
$$

We have

$$
\begin{aligned}
\varpi & =\varsigma_{1}^{T} \Pi_{1} \varsigma_{1}+\varsigma_{2}^{T} \Pi_{2} \varsigma_{2} \\
& =\left(\dot{V}\left(x_{c}\right)+\lambda q^{T} q+e^{T} e-r^{2} w^{T} w+\tilde{x}^{T}\left(X_{1} L\right) \widehat{\Theta}\left(L^{T} X_{1}\right) \tilde{x}+\widehat{\phi}^{T}\left(2 \kappa X_{3}\right) \widehat{\phi}\right),
\end{aligned}
$$

where $\widehat{\Theta}$ is defined in (4.20). Let $\zeta=\left(\begin{array}{c}x_{c} \\ w\end{array}\right)$, thus

$$
\varpi=\zeta^{T} \Pi \zeta+\tilde{x}^{T}\left(X_{1} L\right) \widehat{\Theta}\left(L^{T} X_{1}\right) \tilde{x}+\widehat{\phi}^{T}\left(2 \kappa X_{3}\right) \widehat{\phi}
$$

where $\Pi$ is defined by (4.9). If the matrix inequalities, $\Pi_{1}<0, \Pi_{2}<0$, and $\Upsilon_{i}>0$, are satisfied, we have $\varpi<0$, or equivalently,

$$
\zeta^{T} \Pi \zeta<-\tilde{x}^{T}\left(X_{1} L\right) \widehat{\Theta}\left(L^{T} X_{1}\right) \tilde{x}-\widehat{\phi}^{T}\left(2 \kappa X_{3}\right) \hat{\phi}<0,
$$

for nonzero $\tilde{x}$ and $\widehat{\phi}$, which implies that $\Pi<0$. Hence, by Theorem 4.4 , the closed-loop system is quadratically stable with a robust $\mathcal{L}_{2}$-gain measure $\gamma$ is ensured. This completes the proof.

Remark 4.7. It is highlighted that the $\kappa$ is defined to be the decay rate of the estimated sensor function $\widehat{\phi}$ shown in (3.8a)-(3.8d) such that $\lim _{t \rightarrow \infty} e^{\kappa t}\|\widehat{\phi}\|=0$, when $\widehat{\phi} \in \bar{D}$.

Remark 4.8. The ideas of using $x$ to set the block diagonal matrix are from the separation principle, where the observer and control gain are designed independently [20], and the lower triangular matrix structure of $A_{c}$, where the diagonal elements are the major concerns of the design [21]. It is shown later in the next section that the computation of Lyapunov matrix for controller $\left(X_{2}\right)$ and observer $\left(X_{1}\right)$ is independently accomplished. Hence, in contrast to the general conservative issue of LMI due to the use of a single Lyapunov matrix for all design, the implementation of the proposed block diagonal matrix $x$ obtains less conservativeness. To demonstrate the fact, the robust $\mathcal{L}_{2}$-gain measure in the numerical example shows that the indexed number $\gamma$ is much less than the number shown in [12].

Before presenting the synthesis results in the next section, a useful and important lemma will be stated for clarity. 
Lemma 4.9 (Elimination Lemma see [19]). Given $\mathscr{d}=\mathscr{L}^{T} \in \mathfrak{R}^{n \times n}, \mho \in \mathfrak{R}^{n \times m}$, and $U \in \mathfrak{R}^{n \times p}$ with $\operatorname{Rank}(\mathcal{U})<n$ and $\operatorname{Rank}\left(\mathcal{U}^{T}\right)<n$, there exists a matrix $K$ such that

$$
\mathscr{U}+\mho \boldsymbol{K} \mathfrak{U}^{T}+\mathfrak{U} K^{T} \mho^{T}<0
$$

if and only if

$$
\mho_{\perp}^{T} \mathfrak{d} \mho_{\perp}<0, \quad \boldsymbol{u}_{\perp}^{T} \mathfrak{d} u_{\perp}<0,
$$

where $\boldsymbol{v}_{\perp}$ and $\boldsymbol{U}_{\perp}$ are orthogonal complement of $\boldsymbol{U}$ and $\boldsymbol{U}$, respectively; that is, $\boldsymbol{v}_{\perp}^{T} \boldsymbol{v}=0$ and $\left(\boldsymbol{v}_{\perp} \boldsymbol{v}\right)$ are of maximum rank.

\section{Control and Observer Gain Synthesis}

In this section according to the analyzed results shown in the last section, the observer gain, $L$, and control gain, $K$, will be synthesized. The general LMI synthesis problem involves sets of the form $X \in X$ and a list of matrices $A, B, C, L, K, \Gamma$, and $W$ and scalars $v, \kappa$, and $\theta_{i}$. We will conclude the quadratic stability with a robust $\mathcal{L}_{2}$-gain measure $\gamma$ control problem in an convex optimization fashion. We will also specify the details in the following, and the results will be concluded in an algorithm of computation.

Assume $\operatorname{diag}[\phi] \in \boldsymbol{B} \Phi$ and $\tilde{\phi}_{i} \in \boldsymbol{\Delta}$, for all $i$. Given prespecified matrices $A, B, C, W>0$ and scalars $v>0$ and $\kappa>0$. If, according to Theorem 4.6, there exist matrices $X \in X, L, K, \Gamma$, $\Theta>0$ and $\gamma^{2}$ such that $\Pi_{1}<0, \Pi_{2}<0$, and $\Upsilon_{i}>0$, for all $i$, are satisfied, then the closedloop system is quadratically stable with a robust $\mathcal{L}_{2}$-gain measure $\gamma$. Let the matrix $\Pi_{1}$ be decomposed into

$$
\Pi_{1}=\Pi_{1 \_1}+\Pi_{1 \_2}<0,
$$

where

$$
\begin{gathered}
\Pi_{1 \_1}=\left(\begin{array}{ccc}
\Xi_{2}\left(X_{2}\right) & X_{2} B K & 0 \\
(B K)^{T} X_{2} & (\star \star) & -X_{1} L \\
0 & -L^{T} X_{1} & -\gamma^{2} I
\end{array}\right), \\
\Pi_{1 \_2}=\lambda\left(\begin{array}{c}
C^{T} \\
C^{T} \\
0
\end{array}\right)\left(\begin{array}{lll}
C & C & 0
\end{array}\right), \\
(\star \star)=\Xi_{1}\left(X_{1}\right)+C^{T} C+\left(X_{1} L\right) \Theta\left(L^{T} X_{1}\right) .
\end{gathered}
$$

Thus, we have

$$
\varsigma_{1}^{T} \Pi_{1} \varsigma_{1}=\varsigma_{1}^{T} \Pi_{1 \_1} \varsigma_{1}+\varsigma_{1}^{T} \Pi_{1 \_2} \varsigma_{1} .
$$


It is noted that

$$
\varsigma_{1}^{T} \Pi_{12} \varsigma_{1}=\lambda q^{T} q \geq 0
$$

for $\lambda \geq 0$. Next, by $S$ procedure, the requirement of $\varsigma_{1}^{T} \Pi_{1} \varsigma_{1}<0$ is equivalent to

$$
\varsigma_{1}^{T} \Pi_{1 \_1} \varsigma_{1}<0,
$$

which implies that $\Pi_{1_{-} 1}<0$. Hence, by the well-known Schur complement, the matrix $\Pi_{1_{-} 1}<0$ can be rewritten as

$$
\left(\begin{array}{cccc}
\Xi_{2}\left(X_{2}\right) & X_{2} B K & 0 & 0 \\
(B K)^{T} X_{2} & \Xi_{1}\left(X_{1}\right)+C^{T} C & -X_{1} L & X_{1} L \\
0 & -L^{T} X_{1} & -\gamma^{2} I & 0 \\
0 & L^{T} X_{1} & 0 & -\Theta^{-1}
\end{array}\right)<0 .
$$

The inequality (5.8) can be again rewritten as

$$
\mathscr{U}+\mho K \mathfrak{U}^{T}+U K \mho^{T}<0
$$

where

$$
\begin{gathered}
\mathscr{H}=\left(\begin{array}{cccc}
A^{T} X_{2}+X_{2} A & 0 & 0 & 0 \\
0 & \Xi_{1}\left(X_{1}\right)+C^{T} C & -X_{1} L & X_{1} L \\
0 & -L^{T} X_{1} & -\gamma^{2} I & 0 \\
0 & L^{T} X_{1} & 0 & -\Theta^{-1}
\end{array}\right), \\
\mho=\left(\begin{array}{c}
X_{2} B \\
0 \\
0 \\
0
\end{array}\right), \quad \boldsymbol{U}=\left(\begin{array}{l}
I \\
0 \\
0
\end{array}\right) .
\end{gathered}
$$

Next, the orthogonal complement of $\mathcal{U}$ and $\mathcal{U}$ is given by

$$
\mho_{\perp}=\left(\begin{array}{cccc}
X_{2}^{-1} B_{\perp} & 0 & 0 & 0 \\
0 & I & 0 & 0 \\
0 & 0 & I & 0 \\
0 & 0 & 0 & I
\end{array}\right), \quad u_{\perp}=\left(\begin{array}{ccc}
I & 0 & 0 \\
-I & 0 & 0 \\
0 & I & 0 \\
0 & 0 & I
\end{array}\right)
$$

In which $B_{\perp}$ is defined as the orthogonal complement of $B$ and is such that $B_{\perp}^{T} B=0$ and $\left[\begin{array}{ll}B_{\perp} & B\end{array}\right]$ is of maximum rank. Similarly, $\mho$ and $\mathcal{U}$ are defined as orthogonal complement of $V$ and $U$, respectively. 
It is followed by using the well-known Elimination Lemma stated in Lemma 4.9 that the matrix variable, $K$, can be eliminated from the inequality (5.9), which is equivalent to the following two inequalities:

$$
\mho_{\perp}^{T} \mathscr{e} \mho_{\perp}<0, \quad \mathfrak{u}_{\perp}^{T} \mathfrak{d} \mathfrak{u}_{\perp}<0
$$

Substituting (5.10) and (5.12) into (5.13), we have

$$
\mho_{\perp}^{T} \boldsymbol{t}_{\perp}=\left(\begin{array}{cccc}
B_{\perp}^{T}\left(X_{2}^{-1} A^{T}+A X_{2}^{-1}\right) B_{\perp} & 0 & 0 & 0 \\
0 & \Xi_{1}\left(X_{1}\right)+C^{T} C & -X_{1} L & X_{1} L \\
0 & -L^{T} X_{1} & -\gamma^{2} I & 0 \\
0 & L^{T} X_{1} & 0 & -\Theta^{-1}
\end{array}\right)<0,
$$

which is equivalent to

$$
\begin{aligned}
& B_{\perp}^{T}\left(X_{2}^{-1} A^{T}+A X_{2}^{-1}\right) B_{\perp}<0, \\
& \left(\begin{array}{ccc}
\Xi_{1}\left(X_{1}\right)+C^{T} C & -X_{1} L & X_{1} L \\
-L^{T} X_{1} & -\gamma^{2} I & 0 \\
L^{T} X_{1} & 0 & -\Theta^{-1}
\end{array}\right)<0, \\
& \boldsymbol{u}_{\perp}^{T} \boldsymbol{e} \boldsymbol{u}_{\perp}=\left(\begin{array}{ccc}
A^{T} X_{2}+X_{2} A+\Xi_{1}\left(X_{1}\right)+C^{T} C & X_{1} L & -X_{1} L \\
L^{T} X_{1} & -\gamma^{2} I & 0 \\
-L^{T} X_{1} & 0 & -\Theta^{-1}
\end{array}\right)<0 .
\end{aligned}
$$

It is noted that (5.15), (5.16), and (5.17) cannot be solved simultaneously using LMI Toolbox of Matlab due to its nonconvexity in matrix variable, $X_{2}$. We, therefore, propose that $X_{2}^{-1}$ of (5.15) be solved first. It is easy to find $X_{2}$ using $X_{2}^{-1}$. If we let $\widehat{L}=X_{1} L$, then (5.16) and (5.17) are LMIs in variables, $X_{1}, \widehat{L}, r^{2}$, and $\Theta$, which can be solved simultaneously by the LMI Toolbox of Matlab. The rest LMIs considered are $\Pi_{2}<0$ for a prespecified decaying rate, $\kappa>0, v>0$, and matrix $W>0$, we have

$$
\left(\begin{array}{cc}
-\widehat{\Gamma} W-W^{T} \widehat{\Gamma}^{T}+2 \kappa X_{3} & \widehat{\Gamma} \\
\widehat{\Gamma}^{T} & -v I
\end{array}\right)<0, \quad X_{3}>0,
$$

where $\widehat{\Gamma}=X_{3} \Gamma$. And lastly for $\Upsilon_{i}>0$ using Schur complement, we equivalently have

$$
X-\left(\frac{v}{\theta_{i}}\right) C_{q, i}^{T} C_{q, i}>0, \quad i=1, \ldots, l,
$$

where $\Theta=\operatorname{diag}\left(\theta_{1}, \ldots, \theta_{l}\right)$.

Remark 5.1 (step of computation). Now, we can summarize the step of computation.

(1) Find feasible solutions of $X_{2}^{-1}>0$ and thus $X_{2}$ where LMI (5.15) is satisfied. 
(2) Use the computed matrices $X_{2}$ found in step (1), the robust $\mathcal{L}_{2}$-gain performance problem is placed as the following optimization problem:

$$
\text { minimize } \gamma^{2}
$$

subject to (5.16), (5.17), (5.18), and (5.19)

$$
X_{1}>0, \quad X_{3}>0, \quad \Theta>0 .
$$

(3) Reconstruct control gain, $K$, and the matrices found in step (1) and (2) are substituted into the inequality (5.9), which is LMI on one matrix variable, $K$ and can be solved by LMI Toolbox of Mtalab.

\section{Numerical Example}

This example adopted from [12] will be used to illustrate the proposed design with the following parameters:

$$
A=\left(\begin{array}{ccc}
0 & 1 & 0 \\
0 & 0 & 1 \\
6 & -1 & -4
\end{array}\right), \quad B=\left(\begin{array}{l}
0 \\
0 \\
1
\end{array}\right), \quad C=\left(\begin{array}{lll}
1 & 0 & 0 \\
0 & 1 & 0
\end{array}\right)
$$

We will test three cases to verify the superiority of the designed observer-based controller. First, case no. 1: one sensor fault where the conditions for simulation are similar to those in [12], in which the sensor $y_{1}$ is perfectly normal and sensor $y_{2}$ is subject to faults with $50 \%$ reduction in signal strength. That is, by Theorem $3.1, \phi_{1}$ is equal to 1 , and $\phi_{2}$ is allowed to be time varying or nonlinear function and varies between 0 and 0.5 , that is, $0 \leq \phi_{2} \leq 0.5$. In the simulation, we let $\phi_{2}=0.25+0.1 \sin (10 t)$ to examine the design. Using Matlab LMI control toolbox and following the step of computation in Remark 5.1, the matrix, $X_{2}$, is computed as follows:

$$
X_{2}=\left(\begin{array}{lll}
1.5829 & 1.6959 & 0.9610 \\
1.6959 & 3.8158 & 1.6959 \\
0.9610 & 1.6959 & 1.5829
\end{array}\right)
$$

Then we solve optimization problem proposed by (5.20). We find that

$$
\begin{gathered}
X_{1}=10^{7}\left(\begin{array}{ccc}
9.3574 & -7.9408 & -1.4166 \\
-7.9408 & 8.8721 & -0.9313 \\
-1.4166 & -0.9313 & 2.3478
\end{array}\right), \quad L=\left(\begin{array}{cc}
20.0010 & 0.0263 \\
20.0010 & 0.0263 \\
20.0010 & 0.0263
\end{array}\right), \\
K=\left(\begin{array}{lll}
-7.0000 & -1.7000 & 1.3000
\end{array}\right),
\end{gathered}
$$

and the optimal value of $\gamma^{2}$ is 56.0603 . The control gain $K$ places the eigenvalues of matrix $A+B K$ at $\{-1.00,-0.85 \pm j 0.5268\}$. It is of interest that the eigenvalues of $A-L(\operatorname{diag}[\phi]) C$ are placed around $\{-19,-2,-3\}$ for all $\phi_{2}=0.25+0.1 \sin (10 t)$. It is highlighted that $L(\operatorname{diag}[\phi]) C$ 


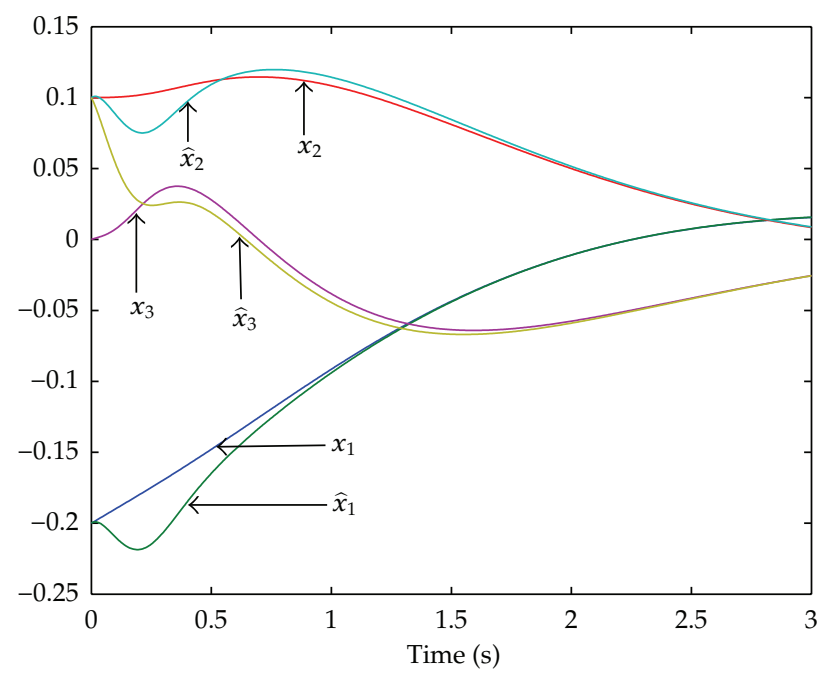

Figure 3: (Case no. 1): The comparison of the plant states, $x$, and observed states, $\hat{x}$, of one sensor fault for the true sensor function $\phi_{2} \in \boldsymbol{B} \Phi$.

intends to closely map the fault sensor signal into its null space. This claim can be verified by viewing

$$
L(\operatorname{diag}[\phi]) C=\left(\begin{array}{lll}
20.0010 \phi_{1} & 0.0263 \phi_{2} & 0 \\
20.0010 \phi_{1} & 0.0263 \phi_{2} & 0 \\
20.0010 \phi_{1} & 0.0263 \phi_{2} & 0
\end{array}\right),
$$

where the effectiveness of $\phi_{2}$ on the eigenvalues of $A-L(\operatorname{diag}[\phi]) C$ has been greatly reduced. Figures 3 and 4 show the complete simulation results. In Figure 3 , the observed states, $\hat{x}$, converge to the plant states, $x$, eventually. In Figure 4 , it is noted that the estimate sensor function $\widehat{\phi}_{1}$ approaches very closely to its true value. However, for fast variation of sensor function $\phi_{2}$, the estimated sensor function, $\widehat{\phi}_{2}$, does not follow it but stay at certain value as $t \rightarrow \infty$. We must highlight that the estimate scheme of sensor function may not be able to approach the true sensor function, see [22] for detail, if lacking of persistent excitation, but to keep the the difference of estimated signal and true sensor function within a bound. It is seen from Figure 9 that $\widetilde{\phi}_{i} \in \Delta, i=1,2$. In Figure 4, the control input, $u$, is also shown. It is worth noting that the smallest possible value of $\gamma^{2}$ obtained by [12] was $416.9764(\gamma=20.42)$. Compare with 50.0603, the number of [12] is much larger. The reasons for that are not hard to conduct; the adaptive law and estimate scheme of faults capture the rate of change of faults, while [12] assumed only bounds of all faults.

The second simulation, case \#2, uses the condition, which is similar to the previous case, where true sensor function $\phi_{1}=1$ and the true sensor function $\phi_{2}$ are not in the designed interval, that is, $\phi_{2} \notin \mathbf{B \Phi}$, which makes $\phi_{2}>0.5$ or $\phi_{2}<0$. In the simulation, we let true sensor function, $\phi_{2}=1.5+0.1 \sin (10 t)$. The simulation results can actually be predicted and are similar to the previous case since the computed observer gain $L$ closely maps the second sensor signal into its null space. It is easy to predict that the states, $x$ and $\widehat{x}$, will be similar to Figure 1 and is shown in Figure 5. In Figure 6, the true sensor functions, $\phi_{1}$ and $\phi_{2}$, estimated 


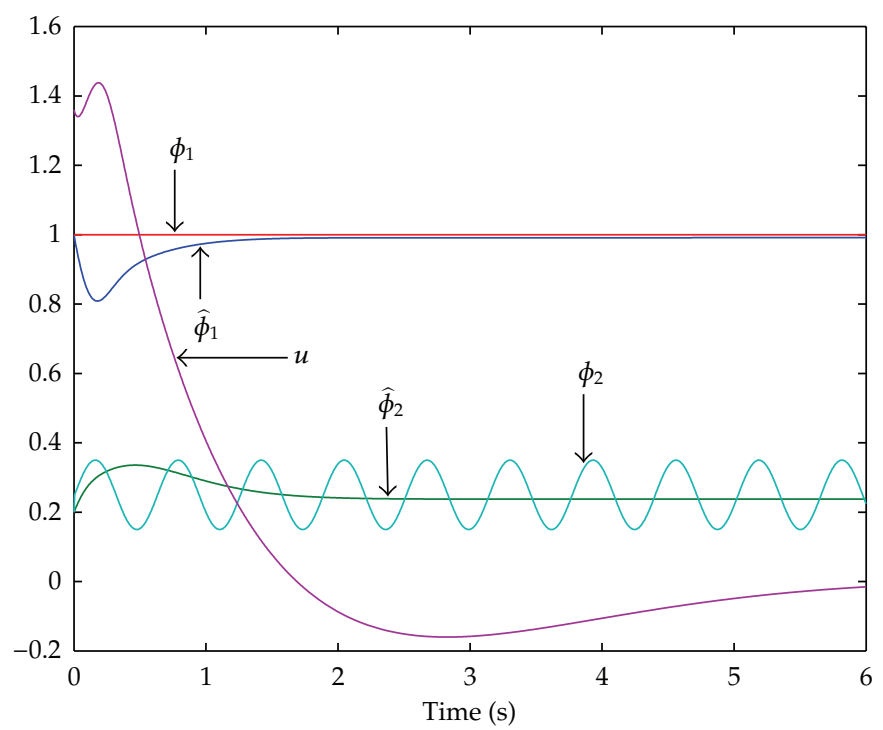

Figure 4: (Case no. 1): True sensor functions, $\phi_{1}=1$ and $\phi_{2}=0.25+0.1 \sin (10 t)$, and estimated sensor functions, $\widehat{\phi}_{1}$ and $\widehat{\phi}_{2}$, for $\phi_{2} \in \boldsymbol{B \Phi}$. The control input, $u$, is also shown.

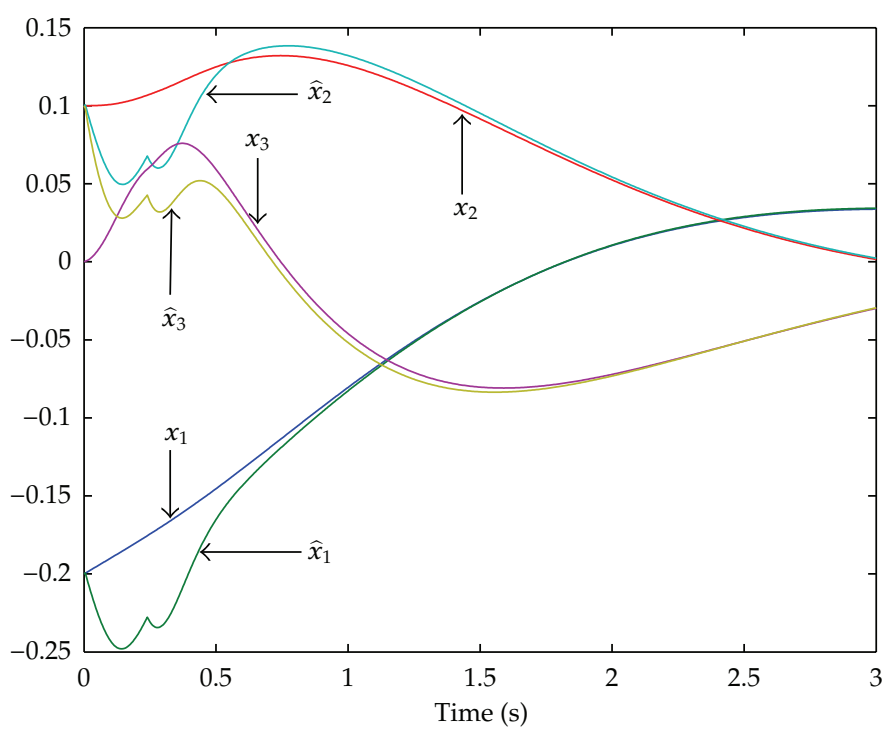

Figure 5: (Case no. 2): The comparison of the plant states, $x$, and observed states, $\hat{x}$, of one sensor fault for the true sensor function $\phi_{2} \notin \boldsymbol{B \Phi}$.

sensor functions, $\widehat{\phi}_{1}$ and $\widehat{\phi}_{2}$, and control signals, $u$, are depicted. It is shown that although the estimated signal, $\tilde{\phi}_{2}$, is within the diagonal norm bound, $\boldsymbol{\Delta}$, which is shown in Figure 6, its results are deteriorated by the poor guess of its initial states.

The third simulation, that is, (case no. 3), is to show two sensor faults. We allow the true sensor function, $\phi_{1}$, shown in Figure 8, to be varying in the pulse form between 0.4 and 0.7 for the first 0.8 second and then $\phi_{1}=0.6+0.03 \sin (10 t), t \geq 0.8$, while keeping $\phi_{2}=0.25+$ 


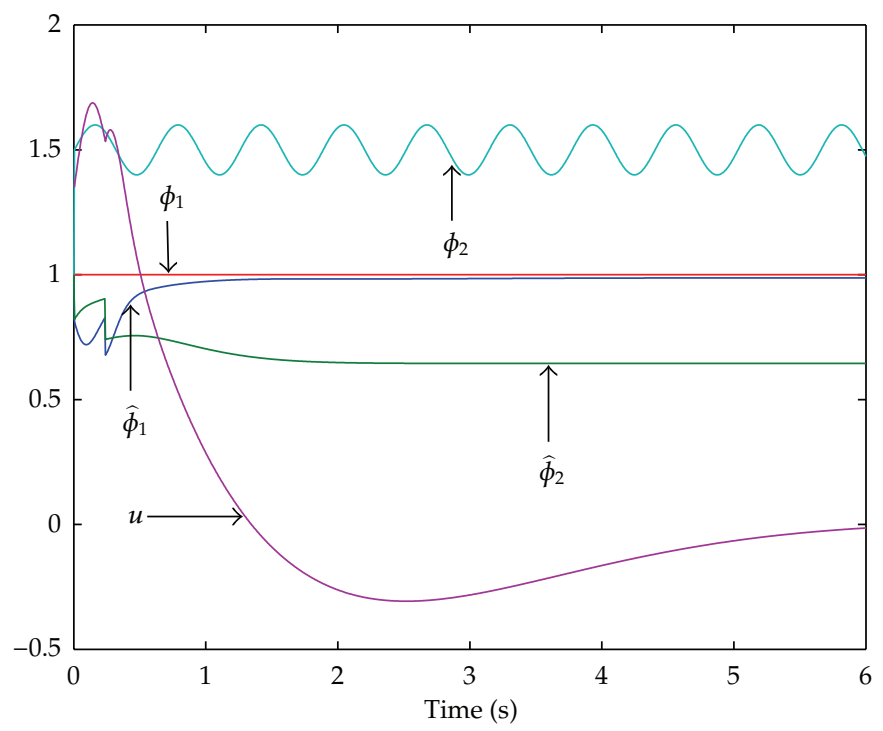

Figure 6: (Case no. 2): True sensor functions, $\phi_{1}=1$ and $\phi_{2}=1.5+0.1 \sin (10 t)$, and estimated sensor functions, $\widehat{\phi}_{1}$ and $\widehat{\phi}_{2}$, for $\phi_{2} \notin \boldsymbol{B \Phi}$. The control input, $u$, is also shown.

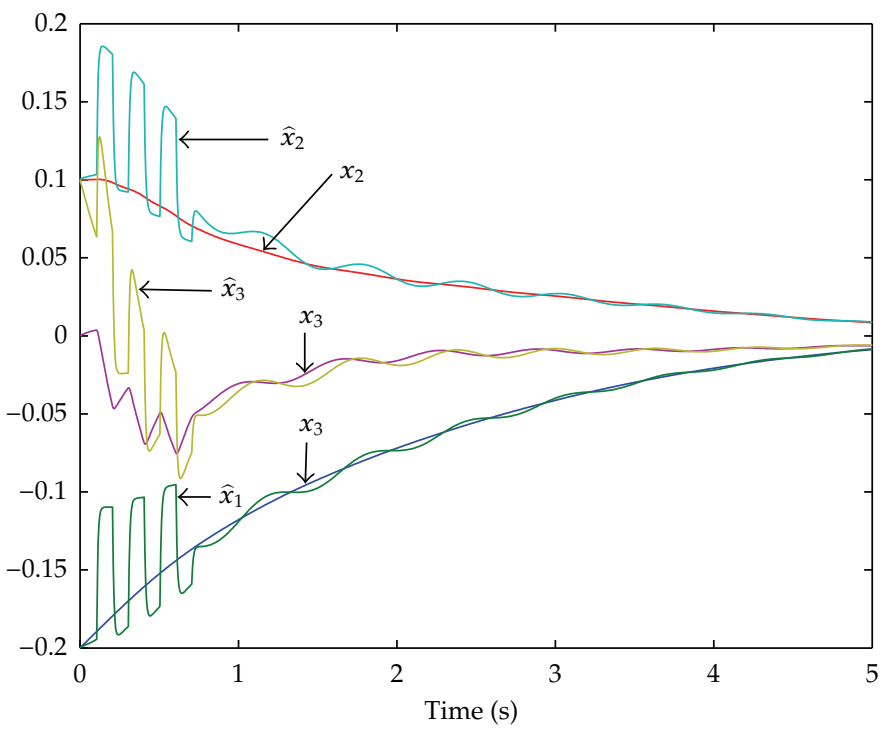

Figure 7: (Case no. 3): The comparison of the plant states, $x$, and observed states, $\hat{x}$, of two sensor fault for the true sensor function $\phi_{i} \in \mathbf{B} \Phi, i=1,2$.

$0.1 \sin (10 t)$ for $t \geq 0$. To compute $K$ and $L$, we assume the polytopic bound with $0.4 \leq \phi_{1} \leq 1$ and $0 \leq \phi_{2} \leq 0.5$. We surprisingly found that $K=(-6.9980-1.69651 .3033)$, which is close to the one sensor fault case, and $L^{T}=\left(\begin{array}{ccc}199.9852 & 199.9852 & 199.9852 \\ 0.0333 & 0.0333 & 0.0333\end{array}\right)$. Under two sensor fault cases, we have raised the $\gamma^{2}=222.5654$. It is not surprising to have the result since the signals for feedback are extremely weak. We have shown the comparison of the true states and observed states in Figure 7, where the large ripples of the observed states are produced due to the pulses of the 

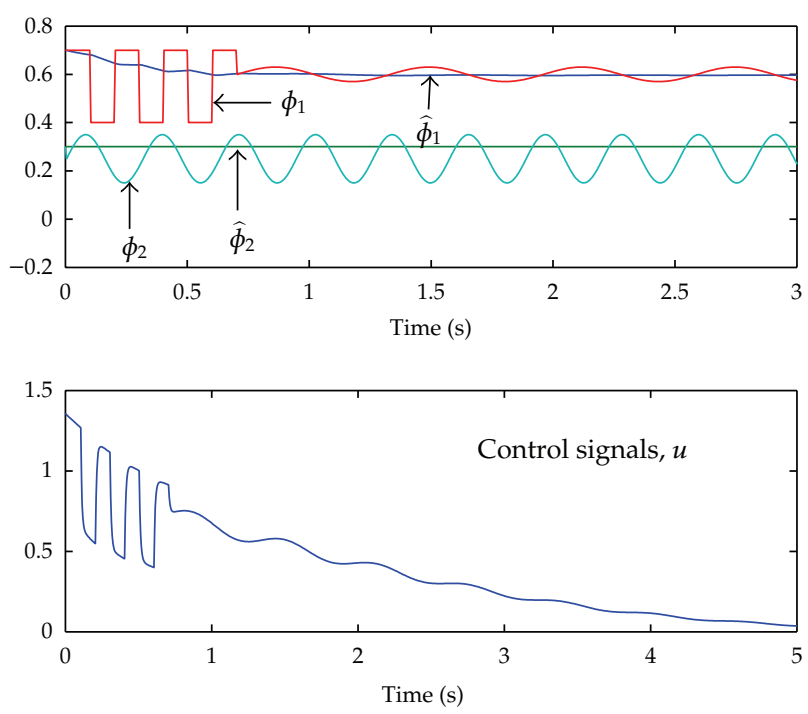

Figure 8: (Case no. 3): The true sensor functions, $\phi_{1}=$ pulses shown in the upper figure for $t \leq 0.8$ and $\phi_{1}=0.6+0.03 \sin (10 t), t \geq 0.8$ and $\phi_{2}=0.25+0.1 \sin (10 t)$, and the estimated sensor functions, $\widehat{\phi}_{1}$ and $\widehat{\phi}_{2}$. The control input, $u$, is also shown.

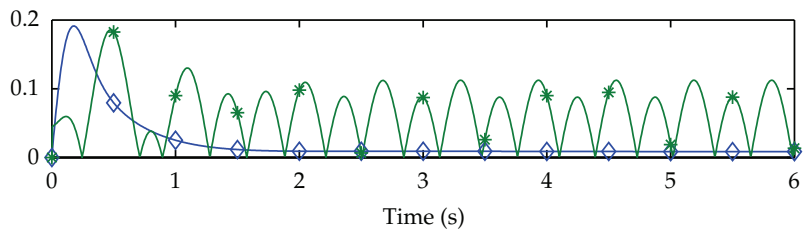

(a)

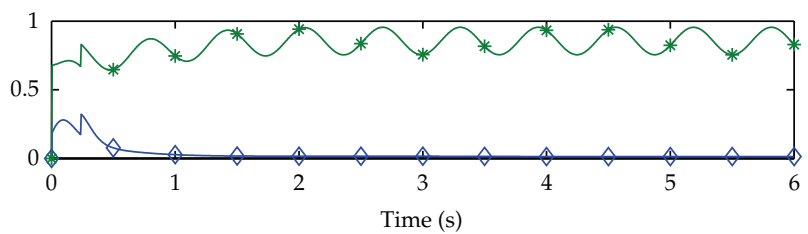

(b)

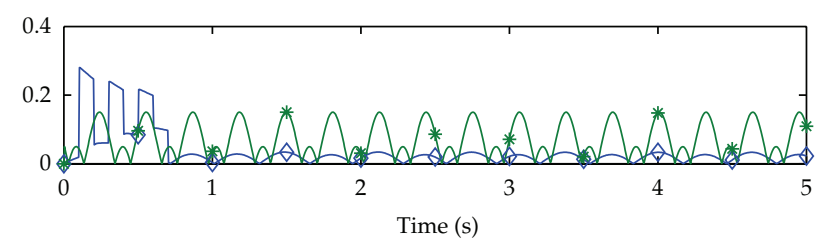

(c)

Figure 9: Solid-diamond line represents $\left|\tilde{\phi}_{1}\right|$, and solid-star line represents $\left|\tilde{\phi}_{2}\right| .(a)$, (b), and (c) are associated with simulation cases 1,2 , and 3, respectively. 
true sensor function in the first 0.8 second but soon died out. In Figure 8, the control input, the true sensor signals, and estimate sensor signals are depicted. We recognize again from Figure 9 that although we assume that $\tilde{\phi}_{i} \in \Delta, i=1,2$, it really depends on the good guess of initial states on the estimate scheme due to viewing the fact that $\widehat{\phi}_{2}$ maintains at its initial states. This, however, can be understood that the associated sensor function is not subject to persistent excitation [22] since it is mapped closely to its null space. Notably, for the third case, the method in [12] failed.

\section{Conclusion}

This paper has developed an observer-based robust control system with an estimate scheme of sensor states against extended bounded-sensor faults. In this design, the control system not only can deal with the sensor fault in a prescribed polytopic bound but also can endure the faults outside the bound. Based on the notion of quadratic stability with a robust $\mathcal{L}_{2}$-gain measure $\gamma$, sufficient conditions for the solvability of the robust control problem have been obtained, and a complete solution was given in terms of LMIs. The numerical example shows the effectiveness of the designed method.

\section{References}

[1] D. Ye and G.-H. Yang, "Adaptive fault-tolerant tracking control against actuator faults with application to flight control," IEEE Transactions on Control Systems Technology, vol. 14, no. 6, pp. 1088-1096, 2006.

[2] M. Blanke, M. Kinnaert, J. Lunze, and M. Staroswiecki, Diagnosis and Fault-Tolerant Control, Springer, New York, NY, USA, 2003.

[3] H. Alwi and C. Edwards, "Robust sensor fault estimation for tolerant control of a civil aircraft using sliding modes," in Proceedings of the American Control Conference, pp. 5704-5709, June 2006.

[4] F. Liao, J. L. Wang, and G. H. Yang, "Reliable robust flight tracking control: an LMI approach," IEEE Transactions on Control Systems Technology, vol. 10, no. 1, pp. 76-89, 2002.

[5] Z. Gao and P. J. Antsaklis, "Stability of the pseudo-inverse method for reconfigurable control systems," International Journal of Control, vol. 53, no. 3, pp. 717-729, 1991.

[6] J. Jiang, "Design of reconfigurable control systems using eigenstructure assignments," International Journal of Control, vol. 59, no. 2, pp. 395-410, 1994.

[7] K. S. Kim, K. J. Lee, and Y. Kim, "Reconfigurable flight control system design using direct adaptive method," Journal of Guidance, Control, and Dynamics, vol. 26, no. 4, pp. 543-550, 2003.

[8] G. Tao, S. M. Joshi, and X. Ma, "Adaptive state feedback and tracking control of systems with actuator failures," IEEE Transactions on Automatic Control, vol. 46, no. 1, pp. 78-95, 2001.

[9] R. J. Veillette, "Reliable linear-quadratic state-feedback control," Automatica, vol. 31, no. 1, pp. 137143, 1995.

[10] Q. Zhao and J. Jiang, "Reliable state feedback control system design against actuator failures," Automatica, vol. 34, no. 10, pp. 1267-1272, 1998.

[11] C.-C. Feng, "Fault-tolerant control and adaptive estimation schemes for sensors with bounded faults," in Proceedings of the 16th IEEE International Conference on Control Applications (CCA '07), pp. 628-633, October 2007.

[12] G. H. Yang, J. L. Wang, and Y. C. Soh, "Reliable $H_{\infty}$ controller design for linear systems," Automatica, vol. 37, no. 5, pp. 717-725, 2001.

[13] K. Zhou and Z. Ren, "A new controller architecture for high performance, robust, and fault-tolerant control," IEEE Transactions on Automatic Control, vol. 46, no. 10, pp. 1613-1618, 2001.

[14] J. Stoustrup and V. D. Blondel, "Fault tolerant control: a simultaneous stabilization result," IEEE Transactions on Automatic Control, vol. 49, no. 2, pp. 305-310, 2004. 
[15] Z. Gao and H. Wang, "Descriptor observer approaches for multivariable systems with measurement noises and application in fault detection and diagnosis," Systems and Control Letters, vol. 55, no. 4, pp. 304-313, 2006.

[16] Z. Gao, S. X. Ding, and Y. Ma, "Robust fault estimation approach and its application in vehicle lateral dynamic systems," Optimal Control Applications and Methods, vol. 28, no. 3, pp. 143-156, 2007.

[17] Z. Gao and S. X. Ding, "Sensor fault reconstruction and sensor compensation for a class of nonlinear state-space systems via a descriptor system approach," IET Control Theory and Applications, vol. 1, no. 3, pp. 578-585, 2007.

[18] Z. Gao and S. X. Ding, "State and disturbance estimator for time-delay systems with application to fault estimation and signal compensation," IEEE Transactions on Signal Processing, vol. 55, no. 12, pp. 5541-5551, 2007.

[19] S. Boyd, L. El Ghaoui, E. Feron, and V. Balakrishnan, Linear Matrix Inequalities in Systems and Control Theory of Studies in Applied Mathematics, vol. 15, SIAM, Philadelphia, Pa, USA, 1994.

[20] F. Franklin, J. D. Powell, and A. Emami-Naeini, Feedback Control of Dynamic Systems, Addison-Wesley, 3rd edition, 1994.

[21] J. C. Geromel, M. C. de Oliveira, and L. Hsu, "LMI characterization of structural and robust stability," Linear Algebra and Its Applications, vol. 285, no. 1-3, pp. 69-80, 1998.

[22] K. J. Astrom and B. Wittenmark, Adaptive Control, Addison-Wesley Longman Publishing, Boston, Mass, USA, 1994. 


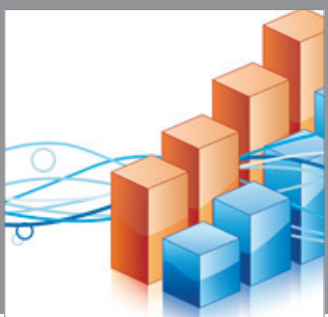

Advances in

Operations Research

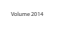

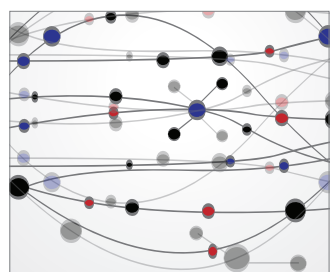

\section{The Scientific} World Journal
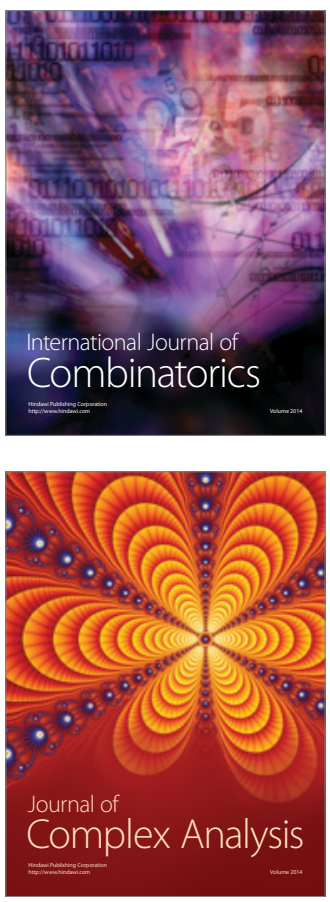

International Journal of

Mathematics and

Mathematical

Sciences
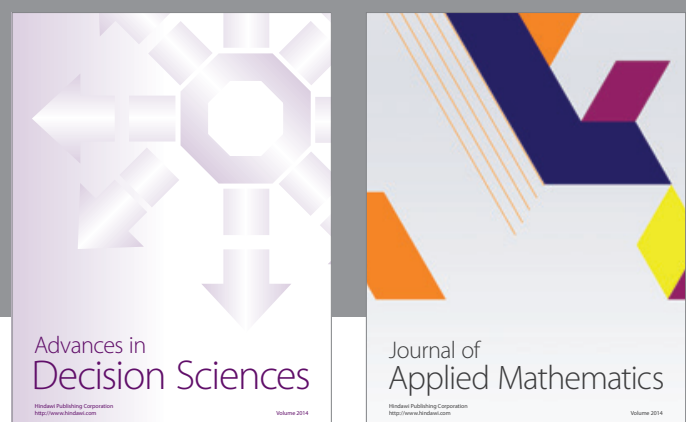

Journal of

Applied Mathematics
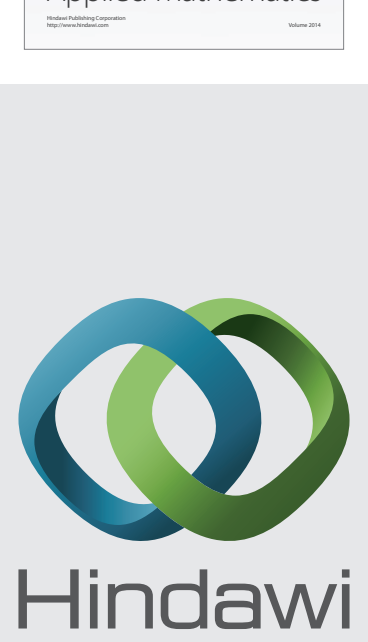

Submit your manuscripts at http://www.hindawi.com
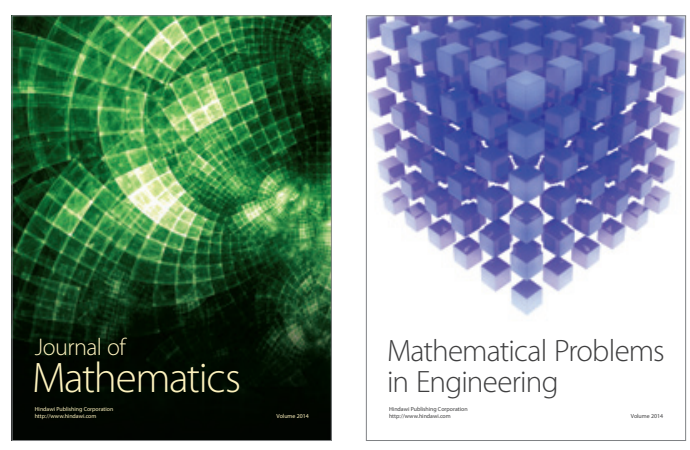

Mathematical Problems in Engineering
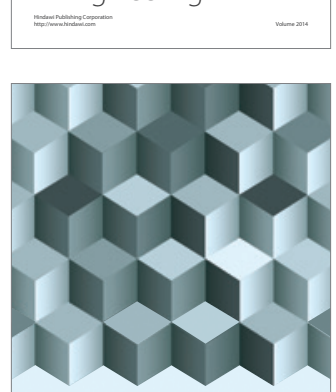

Journal of

Function Spaces
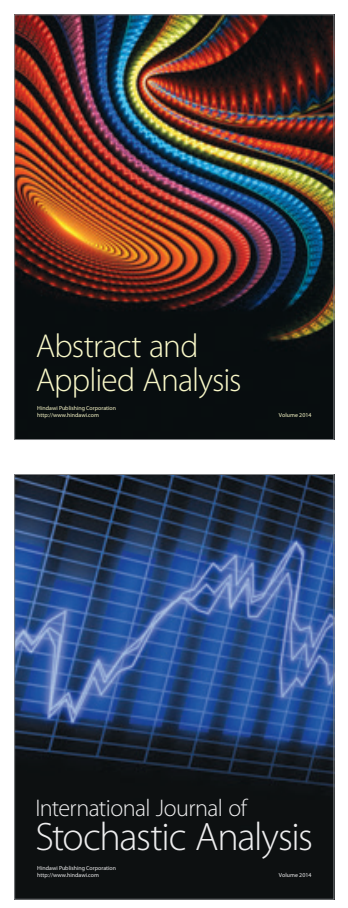

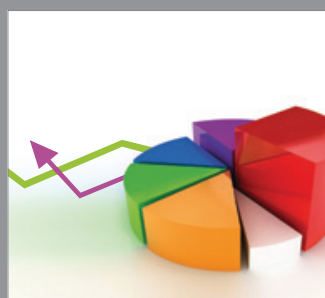

ournal of

Probability and Statistics

Promensencen
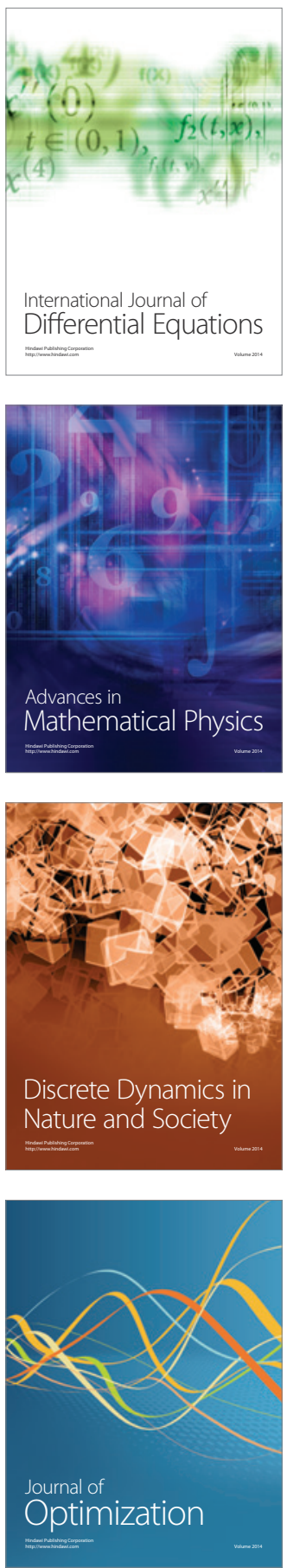